\title{
Block aggregation of stress constraints in topology optimization of structures
}

\author{
J. París, F. Navarrina, I. Colominas \& M. Casteleiro \\ GMNI, Group of Numerical Methods in Engineering, \\ Department of Applied Mathematics, Universidad de A Coruña, \\ E.T.S. de Ingenieros de Caminos, Canales y Puertos, A Coruña, Spain
}

\begin{abstract}
Topology optimization of continuum structures is a relatively new branch of the structural optimization field. Since the basic principles were first proposed by Bendsøe and Kikuchi in 1988, most of the work has been devoted to the so-called maximum stiffness (or minimum compliance) formulations. However, for the past few years a growing effort is being invested in the possibility of stating and solving these kinds of problems in terms of minimum weight with stress (and/or displacement) constraints formulations because some major drawbacks of the maximum stiffness statements can be avoided.

Unfortunately, this also gives rise to more complex mathematical programming problems, since a large number of highly non-linear (local) constraints at the element level must be taken into account. In an attempt to reduce the computational requirements of these problems, the use of a single so-called global constraint has been proposed. In this paper, we create a suitable class of global type constraints by grouping the elements into blocks. Then, the local constraints corresponding to all the elements within each block are combined to produce a single aggregated constraint that limits the maximum stress within all the elements in the block. Thus, the number of constraints can be drastically reduced. Finally, we compare the results obtained by our block aggregation technique with the usual global constraint formulation in several application examples.
\end{abstract}

\section{Introduction}

Topology optimization problems have been usually stated as maximum stiffness (minimum compliance) continuum formulations. However, different approaches 
based on minimum weight formulations with stress constraints have been developed more recently $[1,2,4]$. These minimum weight formulations with stress constraints offer obvious advantages because some difficulties of the minimum compliance formulations can be avoided. In addition, the minimum weight problem states a more realistic problem according to the conventional approaches in other fields of the structural optimization and according to structural norms and laws.

The most usual way of imposing stress constraints in a structural topology optimization problem is to check the stress value on a defined point of each element of the mesh. This approach is usually called the local constraints approach [1,2]. This formulation is very robust but requires a huge computing effort due to the high number of design variables and constraints of this optimization problem.

This huge computing effort forces the treatment of the stress constraints problem from another point of view in order to reduce the required computing resources. Thus, different alternatives to local constraints have been proposed. The most usual way is to impose a global constraint that aggregates all the local constraints [5]. This global constraint approach requires much less computing effort than the local one but the solutions obtained are not so well defined.

Then, we perform a different approach that imposes one global constraint over a group of elements of the mesh that defines a block. This formulation reduces the number of constraints of the problem and consequently the computational effort. The solutions obtained are better defined because the number of elements per constraint is also much smaller in this formulation than in the global approach.

Thus, we compare the solutions obtained with the most usual formulations and with the proposed formulation. In addition, we also compare the computational effort required.

\section{Topology optimization problem}

The minimum weight topology optimization problem can be formulated from a generic point of view as a standard optimization problem. So, the problem can be presented as

$$
\begin{array}{lll}
\text { Find } & \boldsymbol{\rho}=\left\{\rho_{e}\right\} & \\
\text { that minimizes } & F(\boldsymbol{\rho}) & \\
\text { subject to: } & g_{e}(\boldsymbol{\rho}) \leq 0 & e=1, \ldots, N_{e} \\
& 0<\rho_{\min } \leq \rho_{e} \leq 1, & e=1, \ldots, N_{e}
\end{array}
$$

where $\rho_{e}$ is the relative density of the element $e$. The relative density defines the material configuration of the element. Thus, $\rho_{e}=0$ means no material and $\rho_{e}=1$ means solid material. Intermediate values of the relative densities can be analysed according to predefined microstructure models [6]. $F(\boldsymbol{\rho})$ is the objective function (in this case the weight or the cost of the structure) and $g_{e}(\boldsymbol{\rho})$ are the stress constraints. $\rho_{\min }$ and 1 are the corresponding lateral constraints of the optimization problem. The value of $\rho_{\min }$ is usually considered as a small number (0.001) to avoid the singularity of the stiffness matrix. 


\subsection{Objective function}

The minimum cost objective function can be established by different ways. In the literature, several minimum cost objective functions, like the proposed by [1] or [4], can be considered. In this work we have used the minimum cost formulation proposed by [2]. Thus,

$$
F(\boldsymbol{\rho})=\sum_{e=1}^{N_{e}}\left(\rho_{e}\right)^{\frac{1}{p}} \int_{\Omega_{e}} \gamma_{m a t} d \Omega,
$$

where the parameter $p$ is a penalty parameter to avoid intermediate densities in the optimized solution [2]. $\Omega_{e}$ is each element domain and $\gamma_{m a t}$ is the density of the material.

\section{Local constraints formulation}

As we have proposed in the topology optimization problem introduction the constraints of the optimization problem are based on stress approaches. Thus, according to the most usual formulations of topology optimization of structures the stress constraints can be imposed from a local approach point of view.

The local approach of the stress constraints is based on the idea of imposing a stress constraint in a predefined point of each element of the mesh used to solve the structural problem with a Finite Element formulation. According to this stress constraints approach several works have been developed recently [1, 4]. Thus, if we use the Von Mises stress failure criterion in two-dimensional problems each local stress constraint can be considered as:

$$
g_{e}(\boldsymbol{\rho})=\widehat{\sigma}\left(\boldsymbol{\sigma}_{e}^{h}(\boldsymbol{\rho})\right)-\widehat{\sigma}_{\max } \leq 0,
$$

where $\widehat{\sigma}$ is the Von Mises stress and $\boldsymbol{\sigma}_{e}^{h}(\boldsymbol{\rho})$ is the computed stress tensor associated to the element $e$.

The local approach formulation proposed is very robust and produces appropriate results. In addition, the local constraints approach usually presents some additional difficulties (usually called singularity phenomena) which are well known. Some of these difficulties are usually referenced and studied in the literature ([1, 7]). To avoid these difficulties we propose a relaxed formulation to avoid the effects of the singular optimal solutions [3]. Thus,

$$
g_{e}(\boldsymbol{\rho})=\left[\widehat{\sigma}\left(\boldsymbol{\sigma}_{e}^{h}(\boldsymbol{\rho})\right)-\widehat{\sigma}_{\max } \varphi_{e}\right]\left(\rho_{e}\right)^{q} \leq 0,
$$

where

$$
\varphi_{e}=1-\varepsilon+\frac{\varepsilon}{\rho_{e}}
$$

The exponent $q$ can take the values 0 or $1, q=0$ considers real stresses and $q=1$ considers effective stresses in the formulation. The "relaxation parameter" $\varepsilon$ allows to avoid singularity phenomena and usually varies from 0.1 to 0.001 [3]. 


\section{Global constraint formulation}

However, as it can be observed in [3] the local constraints formulation presents an important handicap due to the great computing effort required. This implies important difficulties to solve problems with a high number of elements and, consequently, a high number of non-linear stress constraints.

Due to these computing requirements other formulations have been proposed to reduce the effort of the local constraints approach. A very usual way of reducing these difficulties of the problem is to propose only one global stress constraint that approximates the effect of all the local constraints and simplifies the optimization problem. In this field, several contributions have been proposed with different global functions to approximate the local approach [5]. According to that, we have proposed the use of a global function based on the Kreisselmeier-Steinhauser function. Thus, according to [3] the global constraint can be formulated as:

$$
G_{K S}(\boldsymbol{\rho})=\frac{1}{\mu} \ln \left(\sum_{e=1}^{N_{e}} \exp \left[\mu\left(\frac{\widehat{\sigma}\left(\boldsymbol{\sigma}_{e}^{h}(\boldsymbol{\rho})\right)}{\widehat{\sigma}_{\max } \varphi_{e}}-1\right)\right]\right)-\frac{1}{\mu} \ln \left(N_{e}\right) \leq 0
$$

where the parameter $\mu$ is the aggregation parameter [3] and usually varies from 15 to 40. This formulation reduces a lot the computing effort. However, due to the aggregation of local constraints the optimization algorithms require a higher number of iterations to obtain the optimum solution.

Consequently, the global formulation reduces a lot the computing effort but the solutions obtained are not as reliable as the local approach results. This fact forces us to look for another way of imposing the stress constraints which presents a more reduced effort than the local approach and produces more suitable solutions than the global approach.

The high cost of the local stress constraints approaches has been studied by other authors that propose alternative formulations for the optimization problem that considers local stress constraints but require more reduced computing resources [4].

\section{Block aggregation of stress constraints}

In this paper we propose a different way of solving the problem. The proposed method is a combination of the two ways of imposing stress constraints (local and global approaches). Thus, it is expected to obtain solutions like the ones obtained with the local approach and with a slightly higher computing resource than the required by the global approach.

The proposed technique defines a fixed number of blocks or groups of elements. The number of blocks defines the goodness of the approximation according to the local constraints approach. Once the number of blocks is defined the number of elements of the mesh is distributed equally into these groups. So, all the groups contain, approximately, the same number of elements. 


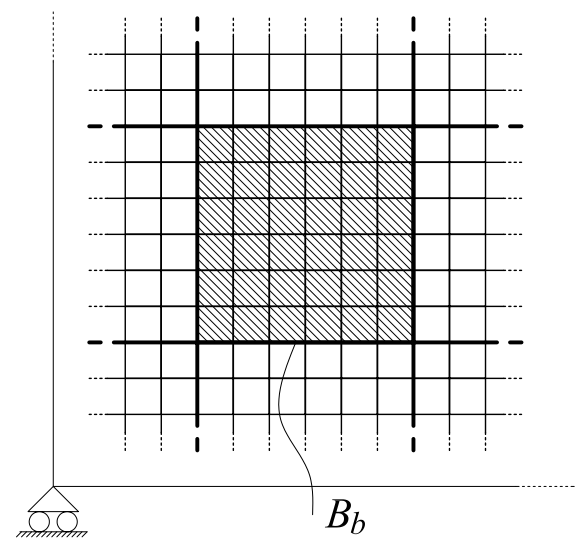

Figure 1: Block definition with the finite element mesh.

The major idea of this approach is to apply over each block of elements $\left(B_{b}\right)$ a global function like the proposed in the global formulation. Thus, the global constraint of the block $b$ is defined as:

$$
G_{b, K S}(\boldsymbol{\rho})=\frac{1}{\mu} \ln \left(\sum_{e \in B_{b}} \exp \left[\mu\left(\frac{\widehat{\sigma}\left(\boldsymbol{\sigma}_{e}^{h}(\boldsymbol{\rho})\right)}{\widehat{\sigma}_{\max } \varphi_{e}}-1\right)\right]\right)-\frac{1}{\mu} \ln \left(N_{e}^{b}\right) \leq 0
$$

where $B_{b}$ is the set of elements of the mesh contained in the block $b$ and $N_{e}^{b}$ is the number of elements contained in the block $b$.

Now, the number of constraints of the optimization problem is equal to the number of blocks. However, the final solutions obtained with this formulation are similar to the results obtained by the local approach because each global constraint presents usually a small number of elements.

In addition, the global function does not suppose an important loss of information in comparison with the local approach. This produces very well defined solutions and the number of iterations required is only a bit higher than the ones required with the local constraints approach.

\subsection{Block definition}

However, this formulation requires to take an important decision. It is very important to decide how to aggregate the elements in blocks. This distribution seems to be very important because the way of aggregating the elements influences the global functions and consequently the global stress constraints.

In this work, we have proposed a very easy way of creating the blocks of elements based on the numbers of the elements in the mesh of Finite Element. Thus, each block contains a number of elements with correlative numbers in the mesh of Finite Elements. This distribution is not generally the most appropriate because it usually produces deformed and long blocks in structured rectangular meshes. 
More compact blocks could be created to obtain even better solutions with different techniques. Random distributions of elements of each block could be also possible. This is a line of research to be developed in the future.

However we have observed in the numerical examples that the block distribution has only a slight influence in the final solutions. It is much more important the number of blocks and the number of elements in each block. It is also very important to define an appropriate value for the parameter $\mu$ of the global constraint of each element. We have tested different meshes and, consequently, different block configurations, obtaining very similar results.

\subsection{Numerical aspects and computing resources}

The proposed formulation presents very important computing advantages. The block aggregation produces an important reduction of the number of constraints which makes the optimization algorithms to work much faster.

We have used a Sequential Linear Programming algorithm with Quadratic Line Search. A more detailed explanation of this optimization algorithm can be found in [2] or [8]. This algorithm produces appropriate results for this problem even with a high number of design variables.

In addition, the number of iterations required by the algorithm is usually more reduced than the required by the global approach, but it is slightly larger than the required with the local approach. However, the computing time of each iteration is much smaller than the required by the local approach. So, an increasing computing speed that allows us to solve bigger problems with smaller computing resources is expected.

\subsection{Sensitivity analysis}

Another important point of the total computing effort required is the sensitivity analysis. The sensitivity analysis usually requires as many calculations as the optimization algorithm and in some cases even more.

The sensitivity analysis of the block aggregated constraints are obtained following the general algorithm proposed in [3]. Thus, full first order derivatives of the constraints are obtained via the adjoint variable method and, once the right search direction is obtained, directional derivatives are obtained by a direct differentiation algorithm. The computing time of the sensitivity analysis is much lower than the required by the local approach. The reduction of the number of constraints allows to compute a smaller number of constraint derivatives for full first order approximation. Directional derivatives also reduces a bit the computing time. However, the directional derivatives require smaller resources than the first order derivatives.

The amount of data storage is also reduced because the number of constraints to obtain derivatives is reduced. Thus the storage of the block aggregated constraints sensitivities require less resources than the derivatives of the local approach. The amount of data storage of the directional derivatives is also reduced. 


\section{Numerical examples}

We present two examples solved with the local approach and with the global approach proposed in order to compare these solutions with the ones obtained with the block aggregated constraints approach that we present. In addition, the computing time and the number of iterations are presented in order to better understand the advantages of the proposed formulation.

The examples solved are two-dimensional structures. However, we show threedimensional solutions, assuming the relative density to be the thickness of each element, to favour the comprehension of the solutions obtained.

\subsection{L-shape beam}

The first example is a L-shape beam $1 \mathrm{~m}$ long and $1 \mathrm{~m}$ high (Figure 2). This beam is supported in the upper edge. Furthermore, a vertical force of $410^{3} \mathrm{kN}$ distributed along two elements is applied in the middle of the right vertical edge. In addition, self-weight is also considered. The beam is made of steel with an elastic limit of $\widehat{\sigma}_{\max }=230 \mathrm{MPa}$ and a Young's Modulus of $E=2.110^{5} \mathrm{MPa}$. The Poisson's ratio is $\nu=0.3$ and the mass density is $\gamma_{m a t}=7650 \mathrm{~kg} / \mathrm{m}^{3}$. The relaxation parameter is $\varepsilon=0.01$ and the penalization is $p=4$. As it can be observed, the solutions (figures 3 and 4 ) are very similar to the ones obtained by [1]. The thickness of the structure is $1 \mathrm{~cm}$.

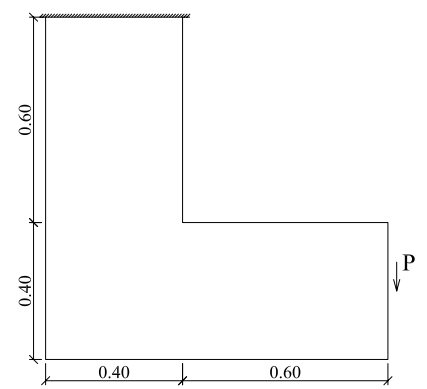

Figure 2: L-shape beam (units in meters).

\subsection{Cantilever support}

The second example is a cantilever support $15 \mathrm{~cm}$ long and $10 \mathrm{~cm}$ high (Figure 5). This structure is supported by two circular holes on the right side. Furthermore, a vertical force of $15 \mathrm{kN}$ is applied in the central hole in the left side. In addition, self weight is also considered. The properties of the material are equal to the ones of the first example, $\varepsilon=0.01$ and the penalization $p=4$. The thickness is $1 \mathrm{~cm}$. 
32 Computer Aided Optimum Design in Engineering X
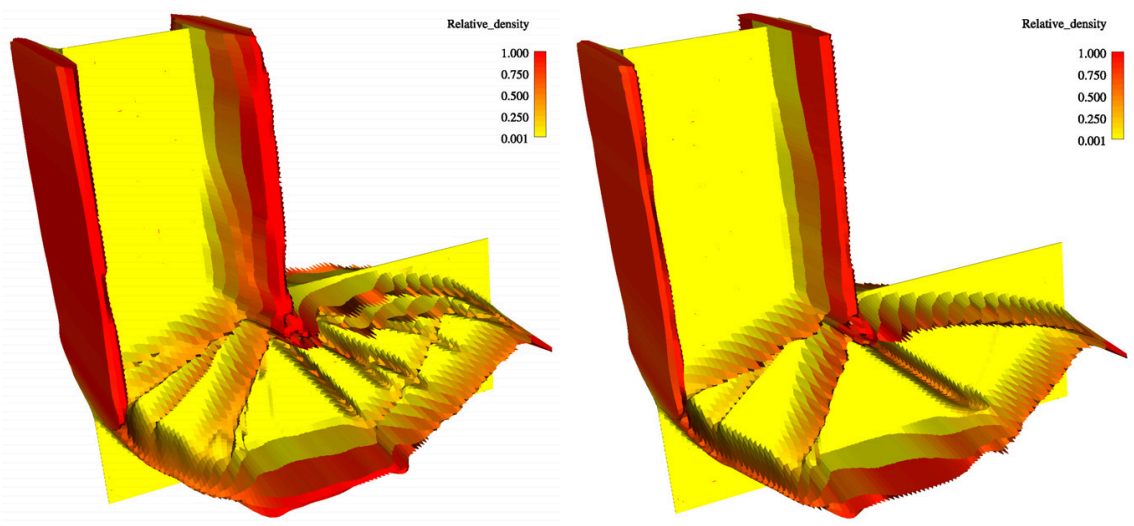

Figure 3: L-shape beam with the local (left) and the global (right) approaches, ( $N_{e}=6400$ (8-node) quadrilateral elements, $q=1, \mu=20, \varepsilon=0.01$ ).

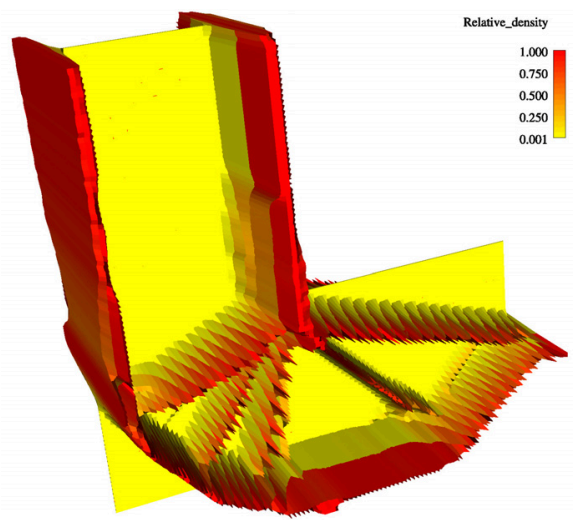

Figure 4: L-shape beam with block aggregation of the stress constraints, (100 blocks with 64 (8-node) quadrilateral elements, $\mu=20, \varepsilon=0.01$ ).

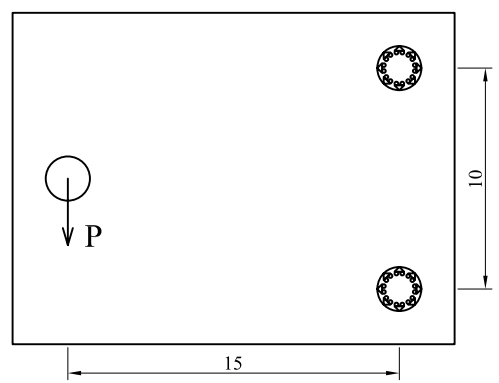

Figure 5: Cantilever support (units in centimeters).

\begin{tabular}{|c|c|c|c|c|c|c|}
\hline & Fig. 3(1) & Fig. 3(r) & Fig. 4 & Fig. 6(1) & Fig. 6(r) & Fig. 7 \\
\hline Comp. time & $8 \mathrm{~d} 20 \mathrm{~h}$ & $7 \mathrm{~h} \mathrm{6} \mathrm{m}$ & $24 \mathrm{~h} 26 \mathrm{~m}$ & $5 \mathrm{~d} 9 \mathrm{~h}$ & $15 \mathrm{~h} 23 \mathrm{~m}$ & $1 \mathrm{~d} \mathrm{1} \mathrm{h}$ \\
\hline Iterations & 152 & 530 & 341 & 253 & 394 & 457 \\
\hline
\end{tabular}



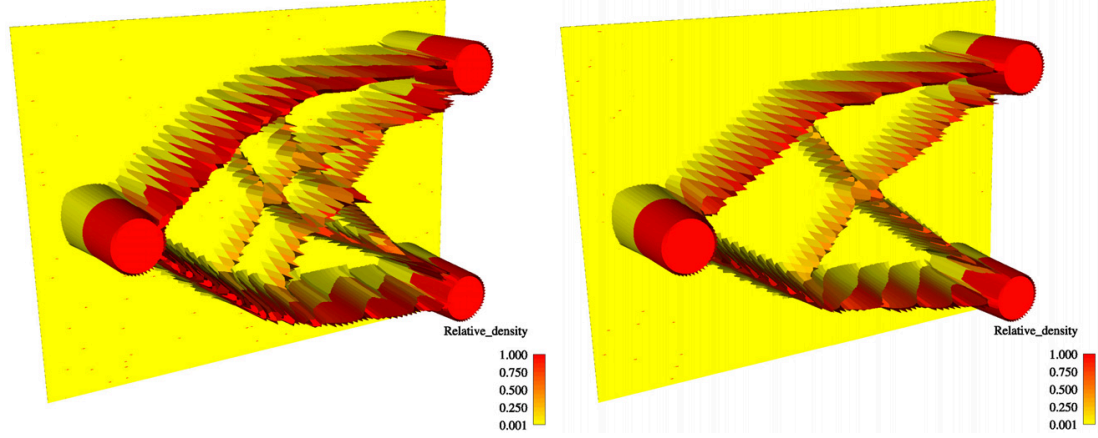

Figure 6: Cantilever support with the local (left) and the global (right) approaches, ( $N_{e}=5808$ (8-node) quadrilateral elements, $q=1, \mu=20, \varepsilon=0.01$ ).

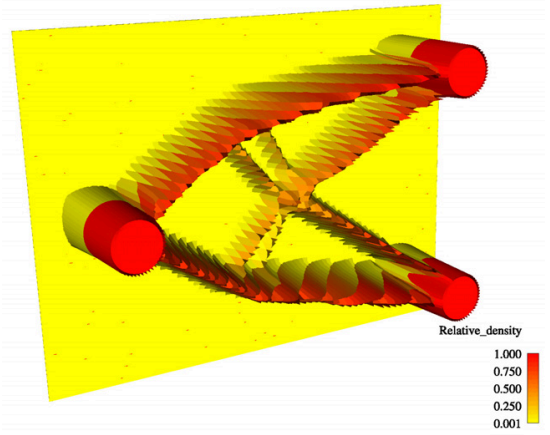

Figure 7: Cantilever support with block aggregated stress constraints, (88 blocks with 66 (8-node) quadrilateral elements, $\mu=20, \varepsilon=0.01$ ).

\section{Conclusions}

- We propose a different technique to consider stress constraints formulations in topological optimization of structures.

- This technique is based on the definition of global constraints applied to groups of elements previously defined.

- This formulation requires much smaller computing resources than the local constraints approach.

- The formulation proposed produces more adequate results than the global constraint approach and with only a small increase in computing resources.

\section{Acknowledgements}

This work has been partially supported by Grant Numbers DPI-2002-00297, DPI2004-05156 and DPI-2006-15275 of the "Ministerio de Educación y Ciencia" of the Spanish Government, by Grant Numbers PGIDIT03-PXIC118001PN and PGIDIT03-PXIC118002PN of the "Dirección Xeral de $I+D$ " of the "Consellería 
de Innovación, Industria e Comercio" of the "Xunta de Galicia", and by research fellowships of the "Universidade da Coruña" and the "Fundación de la Ingeniería Civil de Galicia”.

\section{References}

[1] Duysinx P. and Bendsøe M. P. Topology optimization of continuum structures with local stress constraints, International Journal for Numerical Methods in Engineering, 43, pp. 1453-1478, 1998.

[2] Navarrina F., Muíños I., Colominas I. and Casteleiro M.: Topology optimization of structures: a minimum weight approach with stress constraints, $\mathrm{Ad}$ vances in Engineering Software, 36, pp. 599-606, 2005.

[3] París J., Casteleiro M., Navarrina F. \& Colominas I., Topology optimization of structures with local and global stress constraints, Int. Conference on Computational \& Experimental Engineering and Sciences, ICCES: Miami, 2007.

[4] Burger M. \& Stainko R., Phase-Field Relaxation of Topology Optimization with Local Stress Constraints, IUTAM Symposium on Topology Design Optimization of Structures, Machines and Materials, Springer: Rungsted, 2005.

[5] Duysinx P. \& Sigmund O., New Developments in Handling Stress Constraints in Optimal Material Distributions, 7th AIAA/USAF/NASA/ISSMO Symposium on Multidisciplinary Design Optimization, AIAA: Saint Louis, 1998.

[6] Bendsøe, M.P., Optimization of structural topology, shape, and material, Springer: Heidelberg, 1995.

[7] Cheng G. D. \& Guo X., $\varepsilon$-relaxed approach in structural topology optimization, Structural Optimization, 13, pp. 258-266, 1997.

[8] París J., Muíños I., Navarrina F., Colominas I. \& Casteleiro M., A minimum weight FEM formulation for Structural Topological Optimization with local stress constraints, Proceedings of the VI World Congress on Structural and Multidisciplinary Optimization WCSMO6, ISSMO: Rio de Janeiro, 2005.

[9] Navarrina, F. \& Casteleiro, M., A general methodologycal analysis for optimum design, International Journal for Numerical Methods in Engineering, 31, pp. 85-111, 1991. 